\title{
Emergência e crescimento inicial de plântulas de biribá (Rollinia mucosa (Jacq.) Baill) (Annonaceae) em diferentes substratos
}

\section{Emergence and initial growth of seedlings of Rollinia mucosa (Jacq.) Baill (Annonaceae) in different substrates}

\author{
Maria das Graças Rodrigues Ferreira ${ }^{1 *}$; Maurício Reginaldo Alves dos Santos ${ }^{1}$; \\ Edna de Oliveira Silva²; Edilma Pereira Gonçalves ${ }^{3}$; \\ Edna Ursulino Alves ${ }^{4}$; Riselane de Lucena Alcântara Bruno ${ }^{4}$
}

\begin{abstract}
Resumo
O objetivo desse trabalho foi testar a influência de diferentes substratos na emergência e no crescimento inicial de plântulas de biribá. As sementes foram escarificadas na região oposta à de emissão da radícula e, em seguida, semeadas em diferentes substratos: areia lavada $\left(T_{1}\right)$; vermiculita $\left(T_{2}\right)$; bioplant ${ }^{\circledR}\left(T_{3}\right)$; plantmax ${ }^{\circledR}\left(\mathrm{T}_{4}\right)$; areia lavada + vermiculita 1:1 $\left(\mathrm{T}_{5}\right)$; areia lavada + bioplant ${ }^{\circledR} 1: 1\left(\mathrm{~T}_{6}\right)$; areia lavada + plantmax ${ }^{\circledR}$ 1:1 $\left(\mathrm{T}_{7}\right)$; areia lavada + vermiculita 3:1 $\left(\mathrm{T}_{8}\right)$; areia lavada + bioplant $\left({ }^{\circledR} 3: 1\left(\mathrm{~T}_{9}\right)\right.$; e areia lavada + plantmax ${ }^{\circledR}$ 3:1 $\left(\mathrm{T}_{10}\right)$. Foram avaliados porcentagem de emergência das plântulas, índice de velocidade de emergência e crescimento das plântulas (comprimento e massa seca de raiz e parte aérea). $\mathrm{O}$ delineamento experimental utilizado foi o inteiramente casualizado, com 10 tratamentos, em quatro repetições de 25 sementes. Os dados foram submetidos à análise de variância e a comparação entre as médias foi feita pelo teste de Tukey, a 5\% de probabilidade. De modo geral, constatou-se que os substratos comerciais apresentaram as menores porcentagens e velocidades de emergência, sozinhos ou em combinação com outros substratos, sendo que os maiores valores foram observados com o emprego de areia + vermiculita. Em termos gerais, os quatro substratos avaliados, isolados ou em combinação, não divergiram de forma relevante quanto ao crescimento das plântulas, nos 75 dias subsequentes à semeadura.
\end{abstract}

Palavras-chave: Biribazeiro, fruteira nativa, germinação, propagação

\begin{abstract}
In this research, we evaluated the influence of substrates on emergence and initial growth of Rollinia mucosa seedlings. The seedcoat was scarified opposite the micropylar region and the seeds were sown in different substrates: washed sand (T1), vermiculite (T2), bioplant ${ }^{\circledR}(\mathrm{T} 3)$, plantmax ${ }^{\circledR}(\mathrm{T} 4)$, washed sand + vermiculite 1:1 (T5), washed sand + bioplant ${ }^{\circledR}$ 1:1 (T6), washed sand + plantmax ${ }^{\circledR} 1: 1$ (T7), washed sand + vermiculite 3:1 (T8), washed sand + bioplant ${ }^{\circledR}$ 3:1 (T9) and washed sand + plantmax ${ }^{\circledR}$ 3:1 (T10). We determined the percentage and speed of emergence, and the growth of the seedlings (length and dry mass of root and aerial part). The experimental design was completely randomized with four replications of 25 seeds for each of the ten treatments. The data were submitted to analysis of variance and the averages were compared in the Tukey test. For the percentage and speed of emergence,
\end{abstract}

\footnotetext{
1 Pesquisadores, Dr., Embrapa Rondônia, BR 364 - Km 5,5 - Zona Rural - C. Postal 127, 76815-800 Porto Velho, RO. E-mail: mgraca@cpafro.embrapa.br ; mauricio@cpafro.embrapa.br

2 Mestranda, CCA-UFPB, 58397-000 Areia, PB. E-mail: ednaagronomia@yahoo.com.br

3 Profa. Dra., Universidade Federal Rural de Pernambuco, Departamento de Agronomia, Área de Fitotecnia. E-mail: edilmapg@ hotmail.com

4 Prof ${ }^{a}$ Dr $^{\mathrm{a}}$, CCA-UFPB, 58397-000 Areia, PB. E-mail: ednaursulino@cca.ufpb.br; lane@ccaufpb.br

* Autor para correspondência
} 
it was observed that the commercial substrates had lower values, both when they were tested alone and when they were mixed with other substrates. The sand + vermiculite mixture (T8) showed the best results. For growth, no differences were observed during the period of evaluation ( 75 days).

Key words: Biribazeiro, native fruit, germination, propagation

Dentre os representantes da família Annonaceae, destaca-se o biribá (Rollinia mucosa (Jacq.) Baill), planta nativa das matas pluviais Atlântica e Amazônica, que se desenvolve bem em diferentes habitats e tem o Brasil como centro de origem. $\mathrm{O}$ biribazeiro é uma planta que pode atingir altura média de 8 metros (SIMÃO, 1998) e seu fruto, quando maduro, é de coloração amarela, globoso, composto por diversas partes hexagonais, muito unidas, dando um aspecto característico; sua polpa varia de esbranquiçada a creme, com muitas sementes de cor escura; possui um aroma agradável, podendo pesar até 1,3 $\mathrm{kg}$ (LORENZI, 1998). Os frutos têm grande aceitação popular, sendo consumidos in natura. A planta tem ampla dispersão geográfica e o fruto é conhecido também como biribá do pará, fruta da condessa, biribá de pernambuco, pinha, anona e jaca de pobre (COSTA; MULLER, 1995).

As anonáceas, em geral, são bastante suscetíveis às diversas podridões de raiz e colo (KAVATI, 1992), além de serem atacadas por coleobrocas (TOKUNAGA, 2000), o que exige a utilização de porta-enxerto para amenizar os problemas decorrentes. Das anonáceas cultivadas, o biribazeiro parece ser a mais tolerante, com relação ao ataque de pragas, como a broca do coleto (MANICA, 2000) e, também tem demonstrado alguma resistência com relação à podridão-da-raiz ou murcha (JUNQUEIRA et al., 2000).

Como cultivar copa, o biribazeiro pode ser usado para formação de pomares solteiros ou consorciados. Devido ao rápido crescimento e à boa produtividade, essa espécie possibilita retorno econômico no máximo em cinco anos após a implantação do pomar, além de contribuir para a conservação do solo e melhoria das condições ambientais (COSTA; MULLER, 1995).
Apesar do aumento considerável de dados de análise de sementes de espécies nativas, muitas ainda necessitam de informações básicas referentes às condições ideais de germinação. Tal afirmação pode ser verificada nas Regras para Análise de Sementes - RAS (BRASIL, 1992). O conhecimento das condições ideais para a germinação das sementes de uma determinada espécie é de fundamental importância, sendo que os fatores internos como viabilidade e dormência e fatores externos como água, luz, temperatura, oxigênio e a presença de patógenos podem influenciar o processo germinativo (BRASIL, 1992; BEWLEY; BLACK, 1994; CARVALHO; NAKAGAWA, 2000). De acordo com as Regras para Análise de Sementes RAS (BRASIL, 1992), o substrato tem fundamental importância nos resultados de germinação, pois suas características físicas podem influenciar na disponibilidade de água, luz, oxigênio e temperatura às quais as sementes serão submetidas.

Osubstratoéfundamental para odesenvolvimento das raízes, devendo possuir baixa densidade, boa capacidade de absorção e retenção de água, boa aeração e drenagem, o que evita o acúmulo de umidade, além de estar isento de pragas, doenças e substâncias tóxicas (KÄMPF, 2000; WEDLING; GATTO; PAIVA, 2002). Na escolha do material para substrato devem-se considerar o tamanho da semente, sua exigência com relação à umidade, sensibilidade ou não à luz e, ainda, a facilidade que este oferece para o desenvolvimento e avaliação das plântulas (FIGLIOLIA; OLIVEIRA; PIÑARODRIGUES, 1993).

A família Annonacea compreende muitas espécies arbóreas de grande importância como fruteiras, no reflorestamento de áreas degradadas ou mesmo em projetos paisagísticos. Por isso, a 
germinação de sementes e, consequentemente, a produção de plântulas tem sido estudada para várias espécies, como Annona muricata (graviola) (LEDO; CABANELAS, 1997), Xylopia aromatica (Lam.) Mart., X. emarginata Mart. e X. sericea St. Hil. (pindaíba, pimenteira ou pimenta de macaco) (CASTELLANI; DAMINÃO-FILHO; AGUIAR, 2001), Annona squamosa L. (pinheira ou fruta-doconde) (FERREIRA; ERIG; MORO, 2002; KIILL; COSTA, 2003), Annona montana L. (falsa graviola) (OLIVEIRA; ANDRADE; MARTINS, 2005) e a própria $R$. mucosa (SANTOS; ROBERTO; MARTINS, 2005).

O conhecimento do potencial e os fatores que propiciam a germinação das sementes de biribá podem ser considerados um dos pontos de partida para que a espécie venha a ser explorada de maneira racional e sustentável. Diante desses aspectos, o trabalho foi realizado com o objetivo de testar areia, vermiculita, bioplant ${ }^{\circledR}$ e plantmax $\AA$, isolados ou em combinação, como substratos para plantio de sementes de biribá, visando à determinação do substrato mais eficiente para maximizar a porcentagem e a velocidade de emergência e o crescimento inicial de plântulas desta espécie.

Frutos de biribá (Rollinia mucosa) foram colhidos de diferentes árvores matrizes do campo experimental pertencente à Embrapa Rondônia-RO e beneficiados manualmente, sendo suas sementes lavadas em água corrente para retirada da polpa. As sementes foram enviadas para o Laboratório de Análise de Sementes do Centro de Ciências Agrárias da Universidade Federal da Paraíba, em Areia, PB (658'12"'S e 3542'15"W e 574,62 m), onde foram conduzidos os trabalhos, em casa de vegetação. $\mathrm{Na}$ região a temperatura média anual está entre 23-24 ${ }^{\circ} \mathrm{C}$ e precipitação anual é de $1.400 \mathrm{~mm}$.

As sementes de biribá têm dormência devido à impermeabilidade do tegumento à água e, por isso, foram submetidas à escarificação manual com lixa d'água $n^{\circ} 80$ na região oposta à da emissão da radícula. Em seguida, foram semeadas em bandejas plásticas perfuradas no fundo, com dimensões de 29 × $22 \times 10 \mathrm{~cm}$ de comprimento, largura e profundidade, respectivamente. Os seguintes substratos esterilizados em autoclave foram utilizados: areia lavada $\left(\mathrm{T}_{1}\right)$; vermiculita $\left(\mathrm{T}_{2}\right)$; bioplant ${ }^{\circledR}\left(\mathrm{T}_{3}\right)$; plantmax ${ }^{\circledR}\left(\mathrm{T}_{4}\right)$; areia lavada + vermiculita na proporção de 1:1 $\left(\mathrm{T}_{5}\right)$; areia + bioplant ${ }^{\circledR}$ na proporção de $1: 1\left(\mathrm{~T}_{6}\right)$, areia + plantmax $^{\circledR}$ na proporção de 1:1 $\left(\mathrm{T}_{7}\right)$; areia + vermiculita na proporção de $3: 1\left(\mathrm{~T}_{8}\right)$; areia + bioplant ${ }^{\circledR}$ na proporção de 3:1 $\left(\mathrm{T}_{9}\right)$; e areia + plantmax $^{\circledR}$ na proporção de $3: 1$ $\left(\mathrm{T}_{10}\right)$. Após a semeadura as bandejas foram postas em casa de vegetação, com incidência direta da luz solar. Os substratos foram umedecidos diariamente com regadores manuais.

Os tratamentos foram avaliados quanto a:

Porcentagem de emergência - foram utilizadas 100 sementes por tratamento, divididas em quatro repetições de 25 unidades. As contagens do número de plântulas emergidas iniciaram-se aos 28 dias e estenderam-se até os 75 dias após a semeadura, considerando-se como critério de avaliação, as plântulas que apresentavam os cotilédones acima do substrato, sendo os resultados expressos em porcentagem.

Índice de velocidade de emergência (IVE) - realizaram-se contagens diárias das plântulas normais emergidas durante 75 dias, sendo o índice calculado conforme fórmula proposta por Maguire (1962).

Comprimento de plântulas - aos 75 dias após a semeadura, as plântulas normais foram retiradas das bandejas, os cotilédones removidos e foram medidos o comprimento da raiz principal e da parte aérea com o auxílio de uma régua graduada em centímetros, calculando-se o comprimento médio por plântula, em cada repetição.

Massa seca de plântulas - após a contagem final no teste de emergência, as plântulas anteriormente medidas foram submetidas à secagem em estufa regulada a $65^{\circ} \mathrm{C}$ até atingirem peso constante, sendo os resultados expressos em g.plântula ${ }^{-1}$. 
O delineamento experimental utilizado foi o inteiramente ao acaso e os dados obtidos, não transformados, foram submetidos à análise de variância pelo teste $\mathrm{F}$ e as médias comparadas pelo teste de Tukey, a $5 \%$ de probabilidade, utilizando software ESTAT, versão 2.0/2001.

De acordo com os dados da (Tabela 1) observa-se que a porcentagem de emergência foi relativamente baixa para a espécie, isto é, atingiu o valor máximo de $58 \%$, no substrato areia + vermiculita $\left(\mathrm{T}_{5}\right)$. Além disso, pode-se observar que os maiores resultados (acima de 50\% de emergência) foram obtidos apenas nos tratamentos que continham areia e vermiculita, isoladas ou em qualquer das combinações utilizadas, ou seja, nos tratamentos areia $\left(\mathrm{T}_{1}\right)$, vermiculita $\left(\mathrm{T}_{2}\right)$, areia + vermiculita 1:1 $\left(\mathrm{T}_{5}\right)$ e areia + vermiculita 3:1 $\left(\mathrm{T}_{8}\right)$. Os demais tratamentos que continham substratos comerciais, não inertes, isolados ou em combinação resultaram em porcentagens de emergência inferiores a 50\%. Constatou-se também que a porcentagem de emergência de plântulas oriundas de sementes do substrato areia + vermiculita 1:1 $\left(\mathrm{T}_{5}\right)$ foi significativamente superior àquelas obtidas no bioplant ${ }^{\circledR}\left(\mathrm{T}_{3}\right)$, plantmax ${ }^{\circledR}\left(\mathrm{T}_{4}\right) \mathrm{e}$ areia + bioplant $t^{\mathbb{B}}\left(\mathrm{T}_{6}\right)$, e não diferiu estatisticamente dos demais substratos. Resultados diferentes foram obtidos por Cavalcante et al. (2007), que avaliaram a porcentagem emergência de plântulas de araticum (Annona crassiflora Mart.) em solo, solo + areia grossa (em nove diferentes proporções) e areia grossa, isoladamente, e não identificaram diferenças significativas entre os tratamentos. Os autores concluíram que as maiores porcentagens obtidas, de aproximadamente $50 \%$, não são desejáveis para a produção de mudas em um viveiro comercial, onde normalmente se buscam altas porcentagens de germinação em um curto período de tempo.

Tabela 1. Emergência média e índice de velocidade de emergência (IVE) de plântulas de biribá (Rollinia mucosa) oriundas de diferentes substratos. UFPB, Areia-PB, 2009.

\begin{tabular}{|c|c|c|}
\hline Tratamentos & Emergência (\%) & $\begin{array}{c}\text { Índice de velocidade de emergência } \\
- \text { IVE (plântulas } \text { dia }^{-1} \text { ) }\end{array}$ \\
\hline $\mathrm{T}_{1}$ (areia) & $51 \mathrm{ab}$ & $0,31 \mathrm{ab}$ \\
\hline $\mathrm{T}_{2}$ (vermiculita) & $56 \mathrm{ab}$ & $0,30 \mathrm{abc}$ \\
\hline $\mathrm{T}_{3}$ (bioplant $\left.^{\circledR}\right)$ & $36 \mathrm{bc}$ & 0,19 abcd \\
\hline $\mathrm{T}_{4}\left(\right.$ plantmax $\left.^{\circledR}\right)$ & $23 \mathrm{c}$ & $0,14 \mathrm{~cd}$ \\
\hline $\mathrm{T}_{5}($ areia + vermiculita $1: 1)$ & $58 \mathrm{a}$ & $0,32 \mathrm{a}$ \\
\hline $\mathrm{T}_{6}\left(\right.$ areia bioplant $\left.{ }^{\mathbb{B}} 1: 1\right)$ & $28 \mathrm{c}$ & $0,15 \mathrm{bcd}$ \\
\hline $\mathrm{T}_{7}\left(\right.$ areia + plantmax $\left.^{\circledR} 1: 1\right)$ & $38 \mathrm{abc}$ & 0,19 abcd \\
\hline $\mathrm{T}_{8}($ areia + vermiculita $3: 1)$ & $54 \mathrm{ab}$ & 0,26 abcd \\
\hline $\mathrm{T}_{9}\left(\right.$ areia bioplant $\left.{ }^{\circledR} 3: 1\right)$ & $43 \mathrm{abc}$ & 0,19 abcd \\
\hline $\mathrm{T}_{10}\left(\operatorname{areia}+\right.$ plantmax $\left.^{\circledR} 3: 1\right)$ & $38 \mathrm{abc}$ & $0,12 \mathrm{~d}$ \\
\hline
\end{tabular}

Médias seguidas de mesma letra na coluna não diferem estatisticamente entre si, pelo teste de Tukey a $5 \%$.

Os índices de velocidade de emergência repetiram o padrão observado na porcentagem de emergência, isto é, os únicos substratos que apresentaram IVE acima de 0,20 foram, em ordem decrescente, areia + vermiculita $1: 1\left(\mathrm{~T}_{5}\right)$, areia $\left(\mathrm{T}_{1}\right)$, vermiculita $\left(\mathrm{T}_{2}\right) \mathrm{e}$ areia + vermiculita 3:1 $\left(\mathrm{T}_{8}\right)$. Novamente, os valores inferiores foram aqueles que continham substratos não inertes. Os substratos significativamente inferiores a areia + vermiculita 1:1 $\left(\mathrm{T}_{5}\right)$ foram: areia $+\operatorname{plantmax}^{\circledR} 3: 1\left(\mathrm{~T}_{10}\right)$, $\operatorname{plantmax}^{\circledR}\left(\mathrm{T}_{4}\right)$ e areia + bioplant $^{\circledR}\left(\mathrm{T}_{6}\right)$.

De forma geral constatou-se que os substratos comerciais foram responsáveis pelas menores médias para essas variáveis, sozinhos ou em combinação 
com os outros substratos, sendo que os maiores valores foram observados com o emprego de areia + vermiculita. Estes resultados concordam com os de Melo et al. (2005), quando avaliaram o efeito de diferentes substratos na germinação de sementes de angico (Anadenanthera colubrina (Vell.) Brenan), em condições de laboratório, e observaram que os tratamentos com maior porcentagem de sementes germinadas foi com o emprego de areia e vermiculita.

A eficiência da vermiculita na promoção da emergência observada neste trabalho reflete as propriedades físico-químicas deste substrato, que possibilitam uma alta capacidade de retenção de água e condições ideais de aeração, o que a torna adequada para a utilização em ensaios de germinação (ANDRADE et al., 2000). Em trabalho realizado com sementes de jenipapo (Genipa americana L.) utilizando-se a vermiculita, Andrade et al. (2000) verificaram que esse substrato é constituído de partículas maiores, com espaço vazio, menor densidade aparente (ou menor grau de compactação), maior arejamento e, portanto, maior facilidade para emergência das plântulas. Os substratos que proporcionaram as maiores porcentagens de emergência de plântulas provavelmente reuniram características necessárias de um bom substrato para germinação, tais como porosidade e esterilidade. Uma boa porosidade permite o movimento de água e ar no substrato, favorecendo a germinação. Para que isso ocorra, as sementes não necessitam de nutrientes, mas apenas de sua hidratação e aeração para que se procedam as reações que induzam à formação de caulículo e radícula. A esterilidade do substrato é outro fator importante para o aumento na porcentagem de germinação das sementes, não atuando como fonte de patógenos de solo que poderiam afetar a germinação e o estabelecimento das plântulas (SIMÃO, 1971).

$\mathrm{Na}$ (Tabela 2) pode-se observar que as plântulas oriundas de sementes no substrato vermiculita $\left(\mathrm{T}_{2}\right)$ expressaram maior comprimento da raiz principal. O resultado obtido nesse substrato foi significativamente superior ao daqueles tratamentos com areia $\left(\mathrm{T}_{1}\right)$, bioplant ${ }^{\circledR}\left(\mathrm{T}_{3}\right)$, plantmax ${ }^{\circledR}\left(\mathrm{T}_{4}\right)$, areia + vermiculita 1:1 $\left(\mathrm{T}_{5}\right)$ e areia + plantmax $^{\circledR} 3: 1\left(\mathrm{~T}_{10}\right)$ e não diferiram estatisticamente dos resultados dos substratos areia + bioplant $^{\circledR} 1: 1\left(\mathrm{~T}_{6}\right)$, areia + plantmax $^{\circledR}$ 1:1 $\left(\mathrm{T}_{7}\right)$, areia + vermiculita 3:1 $\left(\mathrm{T}_{8}\right)$ e areia + bioplant $^{\circledR} 3: 1\left(\mathrm{~T}_{9}\right)$.

Tabela 2. Comprimento médio e massa seca média da raiz e da parte aérea de plântulas de biribá (Rollinia mucosa) em função de diferentes substratos. UFPB, Areia-PB, 2009.

\begin{tabular}{|c|c|c|c|c|}
\hline \multirow{2}{*}{ Tratamentos } & \multicolumn{2}{|c|}{ Comprimento $(\mathrm{cm})$} & \multicolumn{2}{|c|}{ Massa seca (g plântula ${ }^{-1}$ ) } \\
\hline & Raiz principal & Parte aérea & Raízes & Parte aérea \\
\hline $\mathrm{T}_{1}$ (areia) & $8,05 \mathrm{bc}$ & 4,15 & $0,0565 \mathrm{a}$ & $0,0460 \mathrm{a}$ \\
\hline $\mathrm{T}_{2}$ (vermiculita) & $11,50 \mathrm{a}$ & $5,80 \mathrm{a}$ & $0,0858 \mathrm{a}$ & $0,0895 \mathrm{a}$ \\
\hline $\mathrm{T}_{3}\left(\right.$ bioplant $\left.^{\circledR}\right)$ & $7,70 \mathrm{bc}$ & $4,00 \mathrm{a}$ & $0,0460 \mathrm{a}$ & $0,0383 \mathrm{a}$ \\
\hline $\mathrm{T}_{4}\left(\right.$ plantmax $\left.^{\mathbb{R}}\right)$ & $5,72 \mathrm{c}$ & $3,82 \mathrm{a}$ & $0,0405 \mathrm{a}$ & $0,0245 \mathrm{a}$ \\
\hline $\mathrm{T}_{5}($ areia + vermiculita $1: 1)$ & $7,72 \mathrm{bc}$ & $4,07 \mathrm{a}$ & $0,0525 \mathrm{a}$ & $0,0595 \mathrm{a}$ \\
\hline $\mathrm{T}_{6}\left(\right.$ areia + bioplant $\left.^{\circledR} 1: 1\right)$ & $8,35 \mathrm{abc}$ & $4,17 \mathrm{a}$ & $0,0518 \mathrm{a}$ & $0,0400 \mathrm{a}$ \\
\hline $\mathrm{T}_{7}\left(\right.$ areia + plantmax $\left.^{\circledR} 1: 1\right)$ & $8,32 \mathrm{abc}$ & $4,57 \mathrm{a}$ & $0,0550 \mathrm{a}$ & $0,0510 \mathrm{a}$ \\
\hline $\mathrm{T}_{8}($ areia + vermiculita $3: 1)$ & $9,02 \mathrm{ab}$ & $4,70 \mathrm{a}$ & $0,0560 \mathrm{a}$ & $0,0475 \mathrm{a}$ \\
\hline $\mathrm{T}_{9}\left(\right.$ areia bioplant $\left.^{\circledR} 3: 1\right)$ & $8,42 \mathrm{abc}$ & $3,80 \mathrm{a}$ & $0,0478 \mathrm{a}$ & $0,0398 \mathrm{a}$ \\
\hline $\mathrm{T}_{10}\left(\operatorname{areia}+\right.$ plantmax $\left.^{\circledR} 3: 1\right)$ & $7,40 \mathrm{bc}$ & $3,70 \mathrm{a}$ & $0,0470 \mathrm{a}$ & $0,0355 \mathrm{a}$ \\
\hline
\end{tabular}

Médias seguidas de mesma letra na coluna não diferem estatisticamente entre si, pelo teste de Tukey a 5\%. 
Com relação ao comprimento da parte aérea e massa seca das raízes e da parte aérea não houve diferença significativa entre os tratamentos. Resultados diferentes foram obtidos por Bezerra, Momenté e Medeiros Filho (2004), quando obtiveram maior altura e massa seca da parte aérea de plântulas de moringa (Moringa oleifera Lam.) com o substrato plantmax. Para juazeiro (Ziziphus joazeiro Mart.), o substrato terra vegetal + vermiculita proporcionou maior comprimento e massa seca de plântulas (MONIZ-BRITO; AYALAOSUÑA, 2005).

Assim, a escolha do substrato deve ser realizada em função da disponibilidade de materiais, de suas características físicas e químicas, de seu peso e custo, além da sua formulação (CHAVES, 2000). Além dos aspectos tecnológicos deve-se atentar também para a formação perfeita da plântula e facilidade no transplantio embora ainda se tenha pouca muita informação sobre o tipo de substrato que propicie a melhor formação da muda e desenvolvimento inicial da planta.

Os substratos inertes utilizados neste trabalho - areia e vermiculita, isolados ou em combinação, são mais eficientes que os substratos comerciais plantmax ${ }^{\circledR}$ e bioplant ${ }^{\circledR}$ na promoção da porcentagem e da velocidade de emergência de plântulas de Rollinia mucosa.

Os substratos avaliados, isolados ou em combinação não influenciam o crescimento das plântulas nos 75 dias subsequentes à semeadura.

\section{Referências}

ANDRADE, A. C. S.; SOUZA, A. F.; RAMOS, F. N.; PEREIRA, T. S.; CRUZ, A. P. M. Germinação de sementes de jenipapo: temperatura, substrato e morfologia do desenvolvimento pós-seminal. Pesquisa Agropecuária Brasileira, Brasília, v. 35, n. 3, p. 609-615, 2000.

BEWLEY, J. D.; BLACK, M. Seeds: physiology of development and germination. New York: Plenum Press, 1994. $445 \mathrm{p}$.
BEZERRA, A. M. E.; MOMENTÉ, V. G.; MEDEIROS FILHO, S. Germinação de sementes e desenvolvimento de plântulas de moringa (Moringa oleifera Lam.) em função do peso da semente e do tipo de substrato. Horticultura Brasileira, Brasília, v. 22, n. 2, p. 295-299, 2004.

BRASIL. Ministério da Agricultura e da Reforma Agrária. Regras para análise de sementes. Brasília: SNDA/DNDV/CLAV, 1992. 365 p.

CARVALHO, N. M.; NAKAGAWA, J. Sementes ciência, tecnologia e produção. Jaboticabal: FUNEP, 2000. 588 p.

CASTELlANI, E. D.; DAMIÃO-FILHO, C. F.; AGUIAR, I. B. Caracterização morfológica de frutos e sementes de espécies arbóreas do gênero Xylopia (Annonaceae). Revista Brasileira de Sementes, Pelotas, v. 23, n. 1, p. 205-211, 2001.

CAVALCANTE, T. R. M.; NAVES, R. V.; BRAGA FILHO, J. R.; SILVA, L. B. Influência de substratos e do armazenamento de sementes sobre a emergência e crescimento de plântulas de araticum (Annonaceae). Bioscience Journal, Uberlândia, v. 23, n. 4, p. 11-20, 2007.

CHAVES, J. C. M. Normas de produção de mudas. Fortaleza: EMBRAPA Agroindústria Tropical, 2000. 37 p. (Documentos, n. 41).

COSTA, J. P. C.; MÜLLER, C. H. Fruticultura Tropical: o biribazeiro (Rollinia mucosa (Jacq.) Baill. Belém: EMBRAPA-CPATU, 1995. 35 p. (Documentos, n. 84).

FERREIRA, G.; ERIG, P. R.; MORO, E. Uso de ácido giberélico em sementes de fruta-do-conde (Annona squamos $\mathrm{L}$.) visando à produção de mudas em diferentes embalagens. Revista Brasileira de Fruticultura, Jaboticabal, v. 24, n. 1, p. 178-182, 2002.

FIGLIOLIA, M. B.; OliVEIRA, E. C.; PIÑARODRIGUES, F. C. M. Análises de sementes. In: AGUIAR, I. B.; PIÑA-RODRIGUES, F. C. M.; FIGLIOLIA, M. B. (Coord.) Sementes florestais tropicais. Brasília; ABRATES, 1993. p. 137-174.

JUNQUEIRA, N. T. V.; OLIVEIRA, M. A. S.; RAMOS, V. H. V.; PINTO, A. C. Q.; ICUMA, I. M. Controle de doenças da gravioleira no cerrado. Planaltina: Embrapa Cerrados, 2000. (Comunicado Técnico, n. 31).

KÄMPF, A. N. Produção comercial de plantas ornamentais. Guaíba: Agropecuária, 2000. 254 p.

KAVATI, R. O Cultivo da atemóia. In: DONADIO, L. C.; MARTINS, A. B. G.; VALENTE, J. P. (Ed.). Fruticultura tropical. Jaboticabal: FUNEP, 1992. p. 39-70. 
KIILL, L. H. P.; COSTA, J. G. Biologia floral e sistema de reprodução de Annona squamosa L. (Annonaceae) na região de Petrolina-PE. Ciência Rural, Santa Maria, v. 33, n. 5, p. 851-856, 2003.

LEDO, A. S.; CABANELAS, C. I. L. Superação de dormências de sementes de graviola (Annona muricata L.) Revista Brasileira de Fruticultura, Cruz das Almas, v. 19, n. 3, p. 397-400, 1997.

LORENZI, H. Árvores brasileiras: manual de identificação e cultivo de plantas nativas do Brasil. Nova Odessa: Plantarum, 1998. 384 p. v. 1.

MAGUIRE, J. D. Speed of germination aid in selection and evaluation of seedling emergence and vigor. Crop Science, Madison, v. 2, n. 2, p. 176-177, 1962.

MANICA, I. Frutas nativas, silvestres e exóticas 1: técnicas de produção e mercado: abiu, amora-preta, araçá, bacuri, biribá, carambola, cereja-do-rio-grande, jabuticaba. Porto Alegre: Cinco Continentes, 2000. 327 p.

MONIZ-BRITO, K. L.; AYALA-OSUÑA, J. T. Influência de diferentes substratos na germinação de sementes de
Ziziphus joazeiro Mart., Rhamnaceae. Sitientibus. Série Ciências Biológicas, Feira de Santana, v. 5, n. 2, p. 6367, 2005.

OLIVEIRA, I. V. M.; ANDRADE, R. A.; MARTINS, A. B. G. Influência da temperatura na germinação de sementes de Annona montana. Revista Brasileira de Fruticultura, Jaboticabal, v. 27, n. 2, p. 344-345, 2005.

SANTOS, C. E.; ROBERTO, S. R.; MARTINS, A. B. G. Propagação do biribá (Rollinia mucosa) e sua utilização como porta-enxerto de pinha (Annona squamosa). Acta Scientarum, Maringá, v. 27, n. 3, p. 433-436, 2005.

SIMÃO, S. Manual de fruticultura. São Paulo: Ceres, $1971.530 \mathrm{p}$.

. Tratado de fruticultura. Piracicaba: FEALQ, $\overline{1998.7} 60 \mathrm{p}$.

TOKUNAGA, T. A cultura da atemóia. Campinas: CATI, 2000. 80 p. (Boletim Técnico, n. 233).

WEDLING, I.; GATTO, A.; PAIVA, H. N. Substratos, adubação e irrigação na produção de mudas. Viçosa: Aprenda Fácil Editora, 2002. 166 p. 
